ARTICLE

\title{
Two-Phase Flow Simulation of Gas Entrainment Phenomena in Large-Scale Experimental Model of Sodium-Cooled Fast Reactor
}

\author{
Kei ITO ${ }^{1, *}$, Tomoaki KUNUGI ${ }^{2}$, \\ Hiroyuki OHSHIMA ${ }^{1}$ and Takumi KAWAMURA ${ }^{3}$ \\ ${ }^{1}$ Japan Atomic Energy Agency, 4002 Narita, O-arai, Ibaraki-ken, 311-1393, Japan \\ ${ }^{2}$ Kyoto University, Yoshida, Sakyo, Kyoto, Kyoto-fu, 606-8501, Japan \\ ${ }^{3}$ NESI Inc., 4002 Narita, O-arai, Ibaraki-ken, 311-1313, Japan
}

\begin{abstract}
The authors have been developed a high-precision and high-performance numerical simulation algorithm for gas-liquid two-phase flows to simulate the gas entrainment (GE) phenomena in a large-sized sodium-cooled fast reactor in Japan (JSFR). In the simulation algorithm, the piecewise linear interface reconstruction (PLIC) algorithm is employed for the interface-tracking and physics-basis simulation methods are introduced to enhance the numerical reproducibility of interfacial flows. As a result of validation tests, it was confirmed that the developed simulation algorithm could reproduce interfacial flows, including the GE phenomena in simple experiments. In this paper, the developed simulation algorithm is applied to the GE phenomena in a large-scale water experiment which models the complicated geometrical configurations of the structural components in JSFR. For the numerical simulation, an unstructured mesh with about one million cells are generated and optimized to simulate the interfacial deformations near the structural components which cause vortical flows behind them to induce the GE phenomena. As a result of the unsteady simulation, a typical interfacial dynamic behavior, i.e. the development of a gas core (interfacial dent), is well simulated. Therefore, it is confirmed that the developed simulation algorithm is suitable to evaluate numerically the GE phenomena in JSFR.
\end{abstract}

KEYWORDS: gas-liquid two-phase flow, gas entrainment, volume-of-fluid, fast reactor, PLIC

\section{Introduction}

In the design study of a large-sized sodium-cooled fast reactor in Japan (JSFR), the suppression of the gas entrainment (GE) phenomena at the interface between argon cover gas and sodium coolant in the reactor vessel is very important to establish an economically competitive design with advanced light water reactors. ${ }^{1,2)}$ However, the GE phenomena in JSFR are highly complicated interfacial phenomena which are too difficult to be evaluated theoretically. Therefore, we are developing a high-precision and high-performance numerical simulation algorithm for gas-liquid two-phase flows to evaluate the GE phenomena accurately. ${ }^{3)}$ In the simulation algorithm, one of high-precision volume-of-fluid algorithms, i.e. the piecewise linear interface reconstruction (PLIC) algorithm ${ }^{4}$ ) is employed for the accurate numerical simulations of interfacial dynamic behaviors. In addition, two innovative methods have been introduced to enhance the numerical reproducibility of interfacial flows. One is a rigorously volume-conservative method for the volume fraction transport, which works to achieve physically appropriate volume distributions in the volume-of-fluid algorithm. ${ }^{5)}$ The other is physics-basis simulation methods of two-phase flows near gas-liquid interfaces, in which the physically appropriate mechanical balances at the interfaces are considered. ${ }^{6)}$ It has been confirmed that the PLIC algorithm with the two innovative methods give accurate solutions on the interfacial static and dynamic phenomena in rather simple numerical simulations, e.g. a rising bubble in liquid. However, to simulate the GE phenomena in JSFR accurately, only the employments of these methods are not sufficient and it is necessary to model accurately the complicated geometrical configurations of the system components in JSFR. In our simulation algorithm, this is done by an unstructured mesh scheme in which tetrahedral, prism and hexahedral cells with arbitrary shapes can be utilized. The authors have checked that the simulation algorithm can reproduce simple interfacial phenomena accurately even on unstructured meshes as well as structured meshes. Recently, the simulation algorithm with the unstructured mesh scheme has been applied to the numerical simulation of a simple GE experiment ${ }^{7)}$ and succeeded in reproducing the occurrence mechanism and the onset condition of the GE phenomena in the experiment.

In this paper, the authors apply the developed and validated simulation algorithm to the numerical simulation of the GE phenomena in the 1/1.8-scale partial model of JSFR. ${ }^{1)}$ The GE occurrences in the simulation results are discussed to confirm the applicability of the simulation algorithm to the GE phenomena in JSFR.

*Corresponding author, E-mail: ito.kei@jaea.go.jp

(C) 2011 Atomic Energy Society of Japan, All Rights Reserved. 


\section{General Description of Numerical Simulation Algorithm}

\section{Interface-Tracking Algorithm}

In the volume-of-fluid algorithm, the volume fraction transport equation which is written as

$$
\frac{\partial f}{\partial t}+\vec{v} \cdot \nabla f=0
$$

is solved to track gas-liquid interfaces. Here, $\vec{v}$ is the velocity vector and $f$ is the volume fraction which shows the volume ratio of liquid phase in a computational cell. The PLIC algorithm works to produce highly accurate solutions of the transport equation. Namely, the transports of the volume fraction in the PLIC algorithm are performed in consideration with the locations of gas-liquid interfaces which are reconstructed geometrically from the volume fraction distribution. The PLIC algorithm is efficient to simulate interfacial dynamic behaviors accurately. However, the PLIC algorithm is developed originally on structured meshes and is studied hardly on unstructured meshes. ${ }^{5)}$ Therefore, the authors developed the PLIC algorithm on unstructured meshes. In the development, the interface reconstruction procedures in the PLIC algorithm on unstructured meshes were discussed carefully and determined. In addition, the transport method for the volume fraction was also discussed, and a volume-conservative method which established perfect volume conservation for gas and liquid phases was proposed.

\section{Physical Modeling at Gas-Liquid Interface}

It is well known that unphysical solutions, e.g. spurious currents near a gas-liquid interface, can be obtained in the numerical simulations of interfacial phenomena when the mechanical balance at the interface is not considered appropriately. Therefore, in our simulation algorithm, two physics-basis methods are employed to avoid such unphysical solutions. ${ }^{6}$ ) One is an improved velocity calculation method in which velocity and momentum are defined independently to calculate physically appropriate velocity at an interfacial cell. In this calculation method, the velocities in gas and liquid phases $\left(\vec{v}_{g}\right.$ and $\left.\vec{v}_{l}\right)$ can be defined by means of the velocity $(\vec{v})$ and momentum $(\vec{m})$ at an interfacial cell as

$$
\begin{aligned}
& \vec{v}_{g}=\left(\rho_{l} \vec{v}-\vec{m}\right) /\left\{(1-f)\left(\rho_{l}-\rho_{g}\right)\right\}, \\
& \vec{v}_{l}=\left(\vec{m}-\rho_{g} \vec{v}\right) /\left\{f\left(\rho_{l}-\rho_{g}\right)\right\} .
\end{aligned}
$$

The other is the improved calculation method for the pressure gradient at an interfacial cell. In this calculation method, the physically appropriate mechanical balance between pressure gradient and surface tension at the interfacial cell is considered to calculate the pressure gradient consistent with the surface tension. Namely, the pressure gradient $(\nabla p)$ in an interfacial cell is calculated locally by means of the cell face pressure $\left(p_{f}\right)$ on the cell as

$$
\nabla p=\sum_{f} \vec{A}_{f} p_{f} / V
$$

where $V$ is the volume of the cell and $\vec{A}_{f}$ is the cell face vector which indicate the area and normal direction of a cell face.

\section{Computational Schemes}

In the numerical simulation algorithm, the Navier-stokes equation is solved with the volume fraction transport equation to obtain velocity field. As for the discretization schemes, the first-order Euler, second-order upwind and second-order central schemes are applied to the unsteady, advection and diffusion terms, respectively. For the velocity-pressure coupling, the SMAC method ${ }^{9)}$ is employed and the pressure Poisson equation is solved iteratively by means of the BiCG-stab method.

For the acceleration of the numerical simulation, the parallel computation can be performed by the use of the message passing interface (MPI). For the sub-domains partitioning, the METIS $^{10)}$ developed at the University of Minnesota is utilized to equalize the numbers of cells in each sub-domain and to minimize the numbers of boundary faces on the edges between sub-domains.

\section{Verification and Validation}

As a verification of the developed numerical simulation algorithm, a rising bubble in liquid was simulated under several Eötvös number conditions. The simulation results on a highly-distorted unstructured mesh showed very good agreements with the results on a structured mesh and the experimental data. Therefore, it was confirmed that the developed simulation algorithms for gas-liquid two-phase flows provided highly accurate simulation results of interfacial dynamic behaviors even on unstructured meshes. ${ }^{6}$ )

Then, the simulation algorithm was applied to the numerical simulation of a simple GE experiment. ${ }^{7)}$ The simple GE experiment consists of a square rod and a suction pipe in a rectangular channel. In the rectangular channel, an inlet flow generates a wake behind the square rod when the inlet flow passes through the square rod. The wake becomes a vortical flow as it is advected downward to the region near the suction pipe. Then, the vortical flow interacts with the downward flow towards the suction pipe and the strength of the vortical flow rapidly grows up. Accompanied by the growth of the vortical flow, a gas core (an interfacial dent) is generated on the gas-liquid interface and elongated towards the suction pipe. Finally, when the gas core reaches to the mouth of the suction pipe, the gas is entrained into the suction pipe (occurrence of the GE). In the numerical simulation, an unstructured mesh with hexahedral cells is employed. Since the GE phenomena occur in the region near the suction pipe, fine cells are applied to that region for the accurate numerical simulation. The numerical simulation was performed under the GE condition, i.e. the condition under which the GE phenomena were observed in the experiment. Figure 1 shows the simulation result of the interfacial shape (the occurrence of the GE). As shown in this figure, the developed simulation algorithm succeeds in reproducing the 


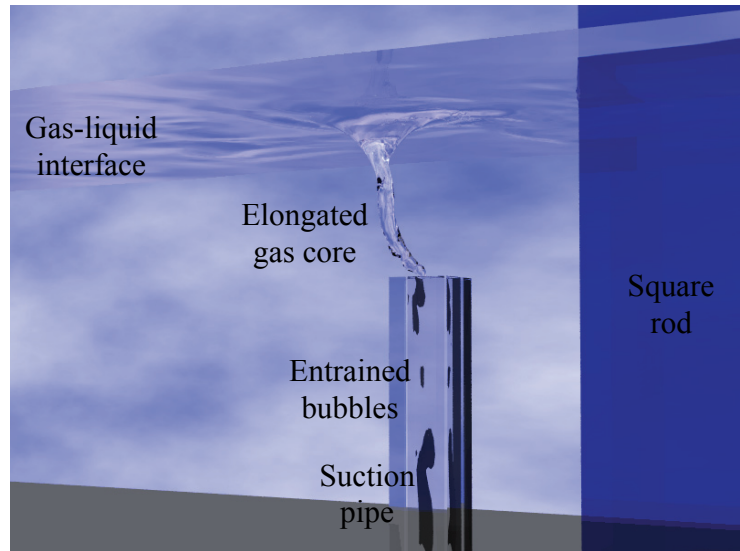

Fig. 1 Simulation result of the simple GE experiment

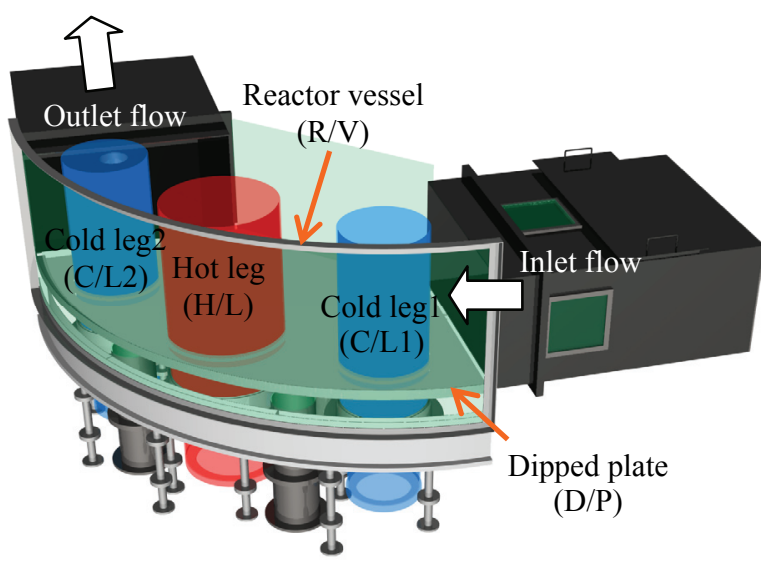

Fig. 2 1/1.8-scale partial model of JSFR

GE occurrence observed in the experiment under the same condition. In addition, it was confirmed that the occurrence mechanism of the GE phenomena in the simulation result was the same as that in the experiment. ${ }^{3)}$ Therefore, the developed simulation algorithm was validated to be capable of reproducing the GE phenomena.

\section{Numerical Simulation of 1/1.8-Scale Partial Model of JSFR}

\section{1/1.8-Scale Partial Model of JSFR}

Figure 2 shows the 1/1.8-scale partial model (1/4-sector model) of JSFR, in which the complicated geometrical configurations of the structural components, e.g. the cold leg $(\mathrm{C} / \mathrm{L})$ and hot leg $(\mathrm{H} / \mathrm{L})$ pipes, are modeled precisely. The radius of the outer wall (reactor vessel) is about $5.5 \mathrm{~m}$ and the widths of the inlet and outlet boundaries are $1.5 \mathrm{~m}$. The diameters of the $\mathrm{C} / \mathrm{L}$ and $\mathrm{H} / \mathrm{L}$ pipes are $0.64 \mathrm{~m}$ and $0.84 \mathrm{~m}$, respectively. In the partial model, the horizontal dipped plate $(\mathrm{D} / \mathrm{P})$, which is employed in JSFR to suppress interfacial fluctuations (the GE occurrences), is installed. As shown in Fig. 3, there are some gaps on the $\mathrm{D} / \mathrm{P}$ and the upward or downward flow is assigned locally to each partial region of the gaps. The experiments are conducted under several inlet velocity, gap velocity and liquid height conditions by the use of water and air as the working fluids.

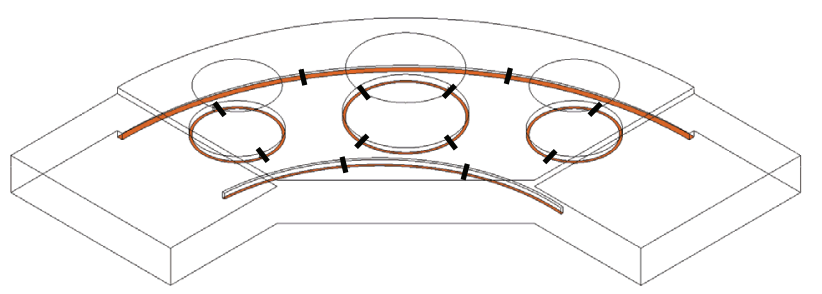

Fig. 3 Gaps between the D/P and system components

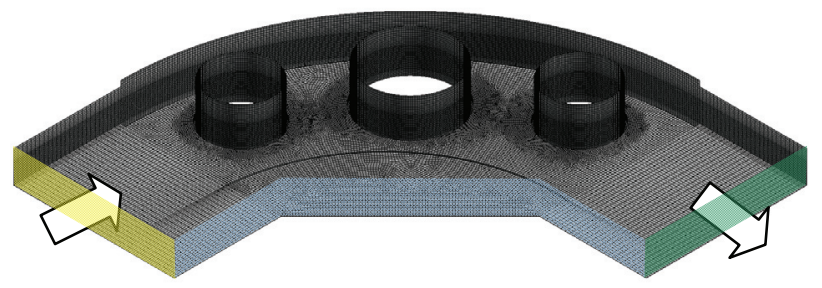

Fig. 4 Unstructured simulation mesh with hexahedral cells

\section{Simulation Conditions}

Figure 4 shows the simulation domain and the simulation mesh. In this unstructured mesh with hexahedral cells, fine cells with the size of about $8.0 \mathrm{~mm}$ are applied to the region near the $\mathrm{C} / \mathrm{L}$ and $\mathrm{H} / \mathrm{L}$ pipes because reproducing the vortical flows near the pipes is a key to simulate the GE phenomena accurately. In addition, fine cells with the size of about $8.0 \mathrm{~mm}$ are also applied to the region near the gas-liquid interface (initial stationary interface) to simulate the deformations of the interface accurately. The other regions are subdivided into about $20-30 \mathrm{~mm}$ cells. The total number of cells is about one million $(1,016,672)$.

The uniform velocity condition is applied to the inlet boundary (the right yellow face in Fig. 4). On the other hand, the outlet boundary (the right green face in Fig. 4) is modeled as a pressure boundary with the hydrostatic pressure distribution. The upper open boundary is treated as the uniform pressure boundary. On each partial region of the gaps between the $\mathrm{D} / \mathrm{P}$ and the structural components, the upward or downward velocity is applied independently based on the measurement data in the $1 / 10$-scale full-sector model experiment of the upper plenum region of JSFR. ${ }^{8)}$ Since it is observed that the GE phenomena do not occur under the rated condition in the $1 / 1.8$-scale partial model and $1 / 10$-scale full-sector model experiments, the numerical simulation is performed under a more severe condition. Namely, the inlet velocity is about $0.092 \mathrm{~m} / \mathrm{s}$ which is 8 times larger than the rated value. In addition, the gap velocities through the $\mathrm{D} / \mathrm{P}$ are also enlarged four times from the rated velocities and the initial water height from the $\mathrm{D} / \mathrm{P}$ is set to $0.2 \mathrm{~m}$ which is about $1 / 4$ of the rated value $(0.805 \mathrm{~m})$. The heights of the inlet and outlet boundaries are the same as the initial water height.

The numerical simulation is performed on Fujitsu Primepower HPC2500 in which each computational node consists of $128 \mathrm{CPUs}$ and the shared physical memory of $512 \mathrm{~GB}$. The simulation domain is partitioned into 64 sub-domains (shown in Fig. 5) and a parallel computation is performed. 


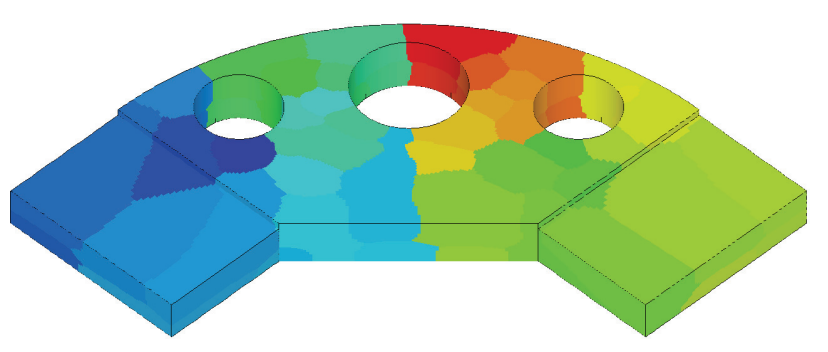

Fig. 5 Domain partitioning for the parallel computation

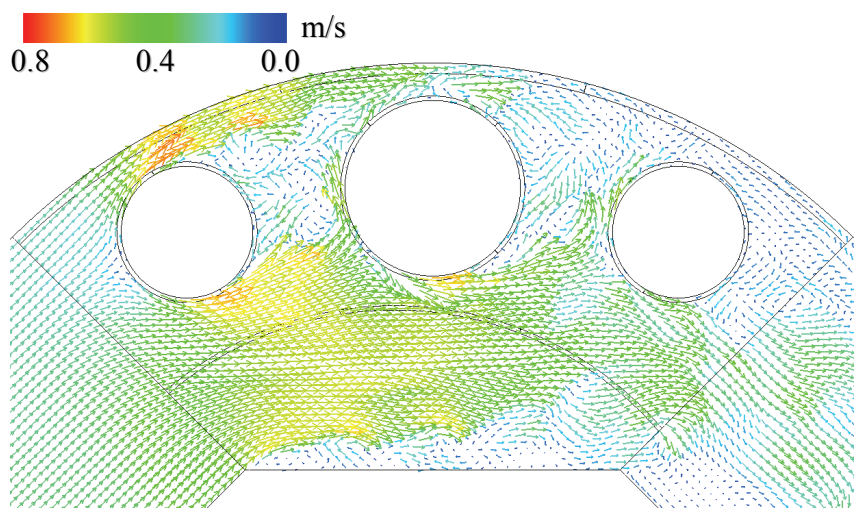

(a) Horizontal velocity

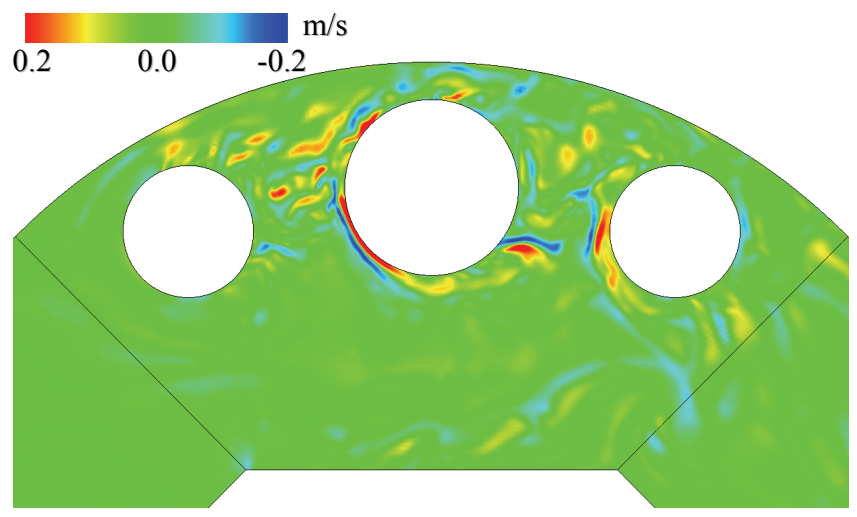

(b) Vertical velocity

Fig. 6 Velocity distributions on a horizontal cross-section

\section{Simulation Results}

(1) Velocity Distribution

In the experiment, the GE phenomena are induced by the vortical flows near the $\mathrm{C} / \mathrm{L}$ and $\mathrm{H} / \mathrm{L}$ pipes. Therefore, the velocity distribution around the pipes should be investigated primarily. Figure 6 shows the fully-developed horizontal and vertical velocity distributions on the horizontal cross-section with the height of $0.18 \mathrm{~m}$ from the $\mathrm{D} / \mathrm{P}$. As for the horizontal velocity distribution in Fig. 6(a), it is observed the inlet flow induces the wake flows behind the $\mathrm{C} / \mathrm{L}$ and $\mathrm{H} / \mathrm{L}$ pipes and vortical flows are formed in the wake flows. Those vortical flows are generated periodically similar to the Karman vortex behind a column. Around the H/L pipe, the upward and downward flows are formed partially as shown

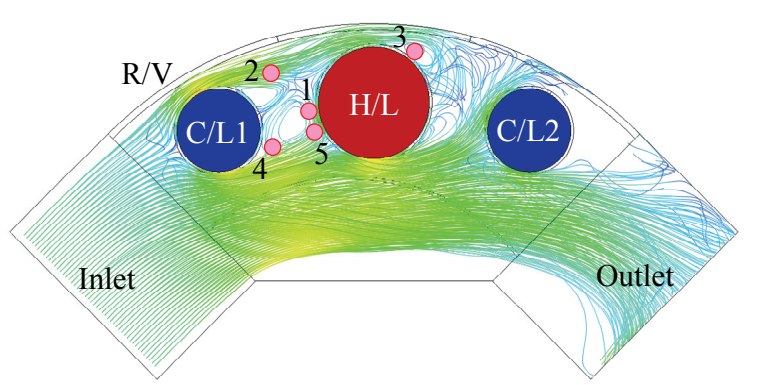

Fig. 7 Time-averaged flow pattern and representative locations of the GE occurrences
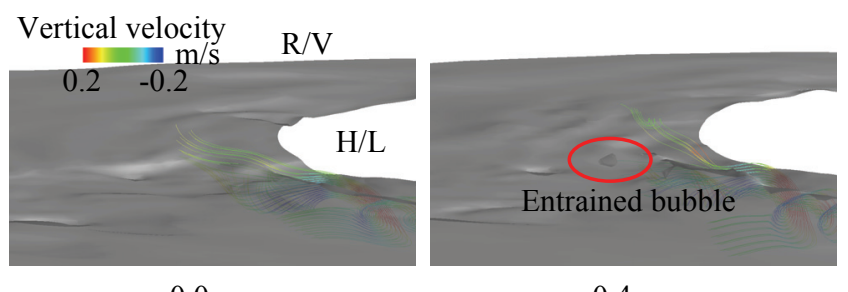

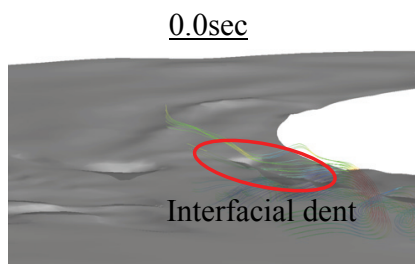

$\underline{0.2 \mathrm{sec}}$

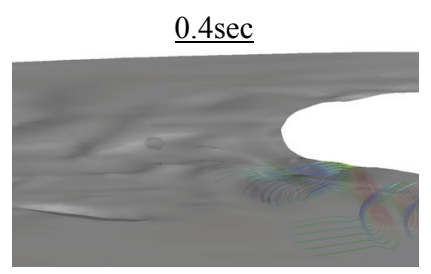

$\underline{0.6 \mathrm{sec}}$
Fig. 8 GE occurrence at the front edge of the $\mathrm{H} / \mathrm{L}$ pipe

in Fig. 6(b). Such a flow pattern is related strongly to the gap flows between the $\mathrm{H} / \mathrm{L}$ pipe and $\mathrm{D} / \mathrm{P}$.

\section{(2) GE Phenomena}

In the simulation results, several GE phenomena are observed. Figure 7 shows the representative locations of the GE occurrences. There are four representative locations in between the $\mathrm{C} / \mathrm{L} 1$ and $\mathrm{H} / \mathrm{L}$ pipes and one at the wake flow region behind the $\mathrm{H} / \mathrm{L}$ pipe. It should be noted that the occurrence of the GE phenomena in the simulation result is determined as a bubble entrainment into water from the interface. The occurrence mechanisms of the GE phenomena in each representative location are somewhat different. Therefore, the authors describe the three typical mechanisms in this paper.

\section{(a) GE Occurrence at Front Edge of H/L Pipe}

At the location 1 in Fig. 7, the inlet flow impinges on the front edge of the H/L pipe. Such a flow makes an upward velocity on the $\mathrm{H} / \mathrm{L}$ wall and a downward flow at the region slightly upstream from the H/L wall (shown in Fig. 6(b)). These oppositely-oriented flows make a shear flow region with a waterfall (submerging flow) which induced the GE phenomena. Figure 8 shows the GE occurrence at this location. In this figure, the dark gray surface shows the gas-liquid interface. It is observed that the interfacial dent is formed near the $\mathrm{H} / \mathrm{L}$ pipe and the bubble with the radius of about $10 \mathrm{~mm}$ is entrained into water by the submerging flow near the dent. However, the entrained bubble rises in water by the buoyancy force and re-emitted though the interface 

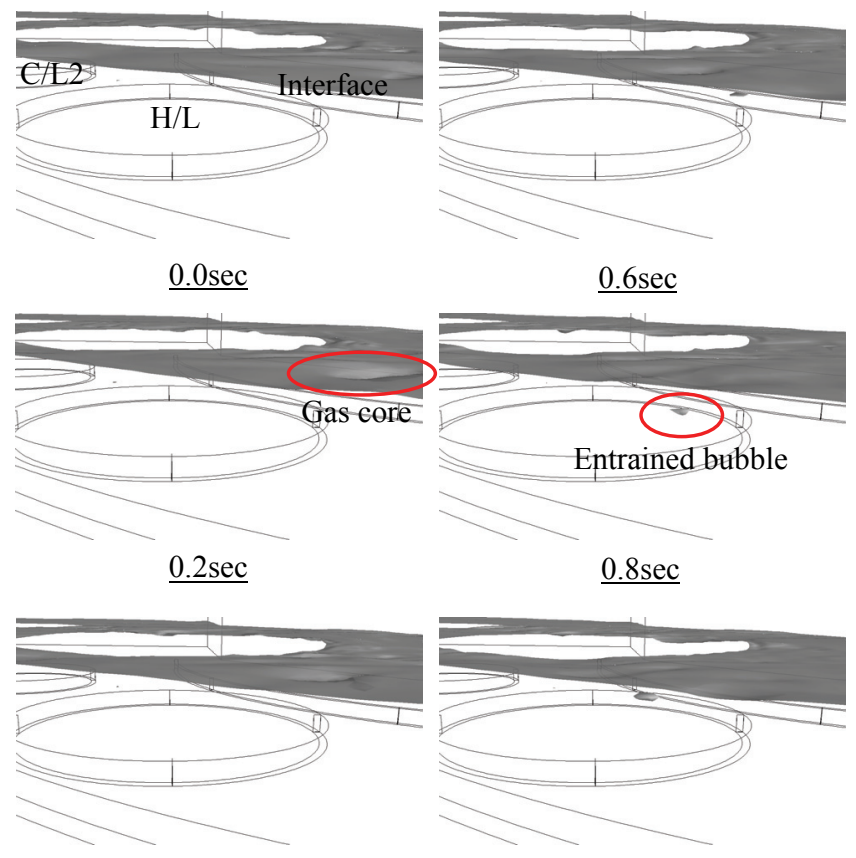

$\underline{0.4 \mathrm{sec}}$

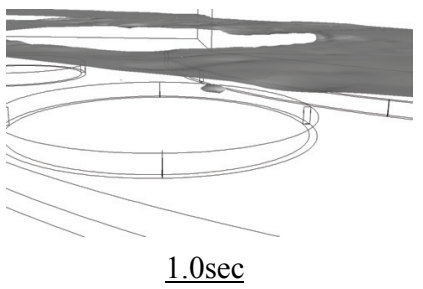

Fig. 9 GE occurrence at the region between the $\mathrm{C} / \mathrm{L} 1$ and $\mathrm{H} / \mathrm{L}$ pipes

within a short period after the entrainment.

(b) GE Occurrence at Region between C/L1 and H/L Pipes

When the inlet flow passes through the $\mathrm{C} / \mathrm{L} 1$, the velocity is highly enhanced in between the $\mathrm{C} / \mathrm{L} 1$ and $\mathrm{R} / \mathrm{V}$ because the flow passage area at the region is small (shown in Fig. 6(a)). Therefore, a strong vortical flow is induced behind the $\mathrm{C} / \mathrm{L} 1$ by the high-speed flow and the GE phenomena are induced at the location 2 in Fig. 7. Figure 9 shows the GE occurrence by such a strong vortical flow. First, the strong vortical flow is intensified further by interacting with the downward flow towards the gap between the H/L pipe and D/P. Then, the gas core is formed on the interface by the strong vortical flow. Finally, the air bubble is pinched-off from the tip of the gas core and entrained into water. Since the similar GE phenomena are observed at the same location in the experiment, it is considered that our simulation algorithm can reproduce the GE phenomena even in a large-scale domain with complicated structural components (as JSFR). The entrained bubble radius is about $18 \mathrm{~mm}$ in this type of the GE phenomena and is much larger than that in the GE phenomena at the front edge of the $\mathrm{H} / \mathrm{L}$ pipe. In addition, the entrained bubble flows into the gap between the $\mathrm{H} / \mathrm{L}$ pipe and $\mathrm{D} / \mathrm{P}$. Therefore, the special attention should be paid to this type of the GE phenomena because they have the potential to entrap air bubbles into the primary circuit of JSFR.

(c) GE Occurrence at Wake Flow Region behind the $\mathrm{H} / \mathrm{L}$ Pipe

A vortical flow is also induced at the wake flow region behind the H/L pipe. Though the induced vortical flow is much weaker than that induced in between the $\mathrm{C} / \mathrm{L} 1$ and $\mathrm{H} / \mathrm{L}$ pipes, it can develop to a very strong vortical flow by interacting with strong downward flows towards the gap

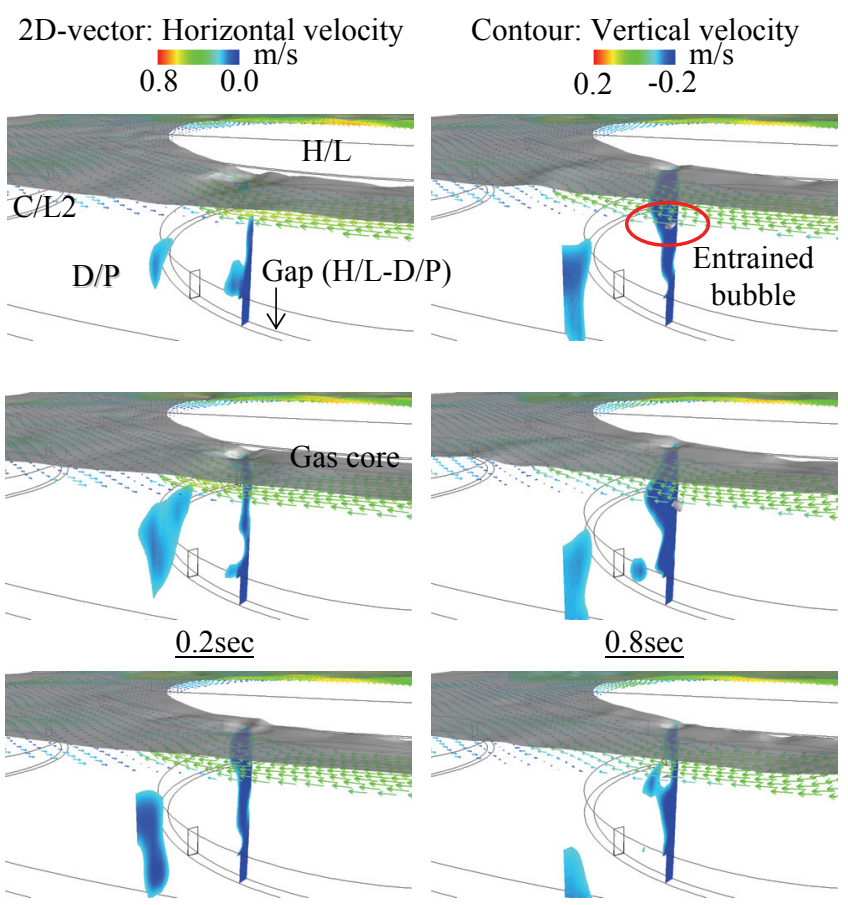

Fig. 10 GE occurrence at the region behind the H/L pipe

between the $\mathrm{H} / \mathrm{L}$ pipe and $\mathrm{D} / \mathrm{P}$. In fact, the downward gap velocity (applied as the boundary condition) is largest at this region. Therefore, as shown in Fig. 10, the gas core develops along the downward flow and the bubble is pinched-off from the tip of the gas core. This type of the GE phenomena is similar to that occurred at the region between $\mathrm{C} / \mathrm{L} 1$ and $\mathrm{H} / \mathrm{L}$ pipes. However, in the former case, the gas core remains at almost the same location in contrast to the latter case where the gas core (vortical flow) is travelling to downstream as shown in Fig. 9. Since such a stable characteristics can lead continuous bubbles entrainment, a special attention has to be paid also to this type of the GE phenomena. The bubble radius is about $14 \mathrm{~mm}$ in this type of the GE phenomena and the entrained bubble flows into the gap between the $\mathrm{H} / \mathrm{L}$ pipe and $\mathrm{D} / \mathrm{P}$ along the downward flow towards the gap.

\section{Conclusion}

In this paper, the numerical simulation algorithm was applied to the GE phenomena in the $1 / 1.8$-scale partial model experiment which modeled the complicated geometrical configurations of the structural components in JSFR. For the numerical simulation, an unstructured mesh with about one million cells were generated and optimized to simulate the GE occurrences near the $\mathrm{C} / \mathrm{L}$ and $\mathrm{H} / \mathrm{L}$ pipes which caused the vortical flows to induce the GE phenomena. In addition, a parallel computing with 64 CPUs was performed to accelerate the numerical simulation. As a result of the unsteady simulation, several types of the GE occurrences are simulated. Among them, the GE occurrence by a mere downward flow is not important because the entrained bubble is re-emitted through the gas-liquid interface by the buoyancy force. However, the GE occurrences induced by strong vor- 
tical flows have to be paid special attention because they can actually entrap the air bubbles into the primary circuit of JSFR. From these simulation results, it can be said that the developed simulation algorithm has the potential to evaluate numerically the GE phenomena in JSFR.

\section{References}

1) N. Kimura, T. Ezure, A. Tobita, H. Kamide, "Experimental study on gas entrainment at free surface in reactor vessel of a compact sodium-cooled fast reactor," J. Nucl. Sci. Technol., 45, 1053-1062 (2008)

2) T. Ezure, N. Kimura, H. Miyakoshi, H. Kamide, "Experimental investigation on characteristic of entrained bubbles due to surface vortex," Proc. the Thirteenth International Topical Meeting on Nuclear Reactor Thermal Hydraulics, Sep. 27Oct. 2, 2009, Kanazawa, Japan (2009), [CD-ROM].

3) K. Ito, T. Kunugi, H. Ohshima, T. Kawamura, "Formulations and validations of a high-precision volume-of-fluid algorithm on non-orthogonal meshes for numerical simulations of gas entrainment phenomena," J. Nucl. Sci. Technol., 46, 366-373 (2009).

4) D. L. Youngs, "Time-dependent multi-material flow with large fluid distortion," Numerical Methods for Fluid Dynamics
(Edited by K. W. Morton, M. J. Baines), American Press, New York 273-486 (1982).

5) K. Ito, Y. Yamamoto, T. Kunugi, "Development of numerical method for simulation of gas entrainment phenomena," Proc. the Twelfth International Topical Meeting on Nuclear Reactor Thermal Hydraulics, Sep. 30-Oct. 5, 2007, Pittsburgh, PA (2007), [CD-ROM].

6) K. Ito, T. Kunugi, “Appropriate formulations for velocity and pressure calculations at gas-liquid interface with collocated variable arrangement," J. Fluid Sci. Technol., 4, 711-721 (2009).

7) K. Okamoto, K. Takeyama, M. Iida, "Dynamic PIV measurement for the transient behavior of a free-surface vortex," Proc. Forth Japan-Korea Symposium on Nuclear Thermal Hydraulics and Safety, Nov. 28 - Dec. 1, 2004, Sapporo, Japan (2004).

8) N. Kimura, K. Hayashi, H. Kamide, M. Itoh, T. Sekine, "Experimental study on flow optimization in upper plenum of reactor vessel for a compact sodium-cooled fast reactor," $\mathrm{Nucl}$. Technol., 152, 210-222 (2005).

9) A. A. Amsden, F. H. Harlow, The SMAC method: a numerical technique for calculating incompressible fluid flows, LA-4370, Los Alamos National Laboratory (LANL) (1970).

10) http://glaros.dtc.umn.edu/gkhome/views/metis/ 